\title{
The balancing act of disclosing outbreaks of SARS-CoV-2 in workplaces
}

\author{
Maxwell Tran BHSc, Adrina Zhong MPH, Lawrence Loh MD MPH
}

Cite as: CMAJ 2021 May 31;193:E806-7. doi: 10.1503/cmaj.210174

W orkplace safety has been an important focus of the COVID-19 pandemic response worldwide. Essential workers, particularly those who cannot work from home and those who are precariously employed (e.g., temporary, contract or agency workers), ${ }^{1}$ are at increased risk for acquiring SARS-CoV-2. Workplaces have been associated frequently with outbreaks of COVID-19 and household transmission of SARS-CoV-2., ${ }^{2,3}$ Policies related to disclosing outbreaks at places of work are both contentious and misunderstood. Should workplace-related outbreaks of COVID-19 be publicly disclosed and, if so, why? We discuss the ethical and public health considerations related to the public identification of workplaces in outbreak.

The Public Health Agency of Canada's Public Health Ethics Consultative Group identified 3 primary public health goals for publicly disclosing surveillance information: helping the public understand COVID-19-related risk to make informed decisions, showing transparency, and building trust by showing that surveillance and response activities are conducted with due diligence. ${ }^{4}$ Public health authorities are further guided by certain ethical principles, which include respect for persons and communities, beneficience, nonmaleficience, justice, trust, transparency, accountability and responsibility. ${ }^{5,6}$

The principles of beneficience and respect for persons and communities dictate that public health authorities should disclose sufficient information about outbreaks for people to make informed decisions about the risks that they are willing to accept, and to subsequently take steps to protect their health. However, the desire for information does not equate a right to access surveillance information, as the rights of people and communities to privacy and freedom from discrimination must also be considered. ${ }^{4}$

Furthermore, the principle of nonmaleficience or "First, do no harm," suggests that public health authorities should actively attempt to mitigate the potential risks and harms associated with workplace-related outbreaks. As such, general public health practice dictates that outbreaks are disclosed only if a public health investigation finds an increased risk to the public from the workplace outbreak, beyond background risk in the community, or if close contacts of workplace-related cases cannot be reached

\section{KEY POINTS}

- When an outbreak of infection at a workplace is identified, standard public health practice is to publicly disclose the name of the workplace only if all contacts cannot be traced and a risk to the public is identified.

- Proponents of naming all workplaces with outbreaks regardless of risk to the public, assert that such a policy would increase transparency on where transmission is occurring, and enable workers and the public to protect themselves.

- However, the rights of people and communities to privacy and freedom from discrimination must be considered paramount when no risk to the public exists.

- Inappropriate disclosure can lead to reputational harm for the affected businesses and risk business owners covering up outbreaks or refusing to cooperate with public health investigations.

- Broad sectoral instead of nominal data for affected workplaces should be used to identify risk and better target interventions, research and advocacy for measures, such as improved worker protections and wage replenishment.

without disclosure. ${ }^{7,8}$ Where the public is not at risk - for example, where a workplace with an outbreak does not permit public access - public health agencies typically do not issue a broad disclosure naming the workplace. ${ }^{7,8}$

In response to calls for increased transparency, arising out of a broader media debate around the availability and detail of data, several health departments have now created more specific criteria for identifying workplace-related outbreaks with public health importance. ${ }^{1}$ Toronto Public Health announced an approach to name workplaces that have outbreaks of substantial duration that are large enough to mitigate privacy concerns, with a high proportion of staff involved in the outbreak and evidence of sustained transmission. ${ }^{9}$ Alberta Health Services reports the names of workplaces with outbreaks involving 10 or more cases, whereas the City of Hamilton Public Health Services reports the names of all workplaces with active outbreaks. ${ }^{1}$ Most recently, the Region of Waterloo Public Health and Emergency Services chose to name workplaces with 50 or more cases, or 15 or more 
cases with $15 \%$ positivity among staff and patrons, and at least 20 employees or patrons; evidence of sustained transmission; and no substantial privacy concerns. ${ }^{10}$

There are many reasons why public health guidance may suggest not publicly naming a workplace that is having a COVID-19 outbreak, the most prominent being people's right to privacy. The disclosure of information that allows people to be identified can have serious consequences, including public shaming and harassment. Not publicly disclosing a work-related outbreak when there is no increased risk to the public protects workers who are positive for SARS-CoV-2 infection and their contacts from stigmatization associated with COVID-19.11 Moreover, businesses that are outed as having an outbreak of infectious disease may face serious consequences that in turn may lead to loss of employment for workers and downstream effects for local economies.

As workplace outbreaks sometimes occur even despite a company's best efforts to comply with precautions, policies that amount to automatic disclosure may prove counterproductive if companies try to cover up outbreaks so as to avoid public shaming. When businesses can trust that disclosures of workplace outbreaks are made with careful consideration and are appropriate and useful, they are more likely to cooperate with efforts of the public health agencies to control an outbreak by allowing rapid access and intervention to protect workers. If businesses delay or decline to cooperate with outbreak investigations because of fear of reputational or economic ruin, both workers and the public will be put at increased risk of infection. In such instances, public disclosure might be warranted if public health units are unable to ascertain the extent of transmission that may be occurring within the facility owing to noncooperation. However, in general, as in the physician-patient relationship, where respect for confidentiality supports trust that ultimately allows the provider to better promote the patient's health, compliance by businesses with directions by public health agencies protects their workers and fosters healthier communities.

Those who call for broader disclosure of workplace-related outbreaks suggest that this information is necessary to assist with research on high-risk sectors and advocacy efforts. Although many public health agencies release summary data on workplace outbreaks by sector, which has led to the identification of high-risk sectors such as manufacturing, distributing/logistics and food processing, ${ }^{3}$ critics have argued that workplace information by sector is sometimes unclear and misleading. Although data may be of low quality, the answer is not public disclosure but rather the improvement of sectoral data quality and integrity to support research, advocacy and government response.

Where a public risk related to a workplace outbreak is identified, broader public disclosure is certainly warranted. However, calls for automatic disclosure of all workplace-related COVID-19 outbreaks distract policy-makers from enacting measures that are likely to protect both essential workers and communities. These essential measures include collaborating with workplaces to implement and enforce infection control protocols, paid sick leave, protection from eviction, wage replenishment, and linguistically and culturally nuanced health and safety information.

\section{References}

1. Moore O, Marotta S. Toronto naming companies with COVID-19 outbreaks. The Globe and Mail 2021 Jan. 4, updated 2021 Jan. 5. Available: https://www.theglobeandmail .com/canada/toronto/article-toronto-naming-companies-with-covid-19-outbreaks/ (accessed 2021 Jan. 10).

2. All Ontario: case numbers and spread. Toronto: Ontario Ministry of Health. Available: https://covid-19.ontario.ca/data (accessed 2021 Jan. 20).

3. Murti M, Achonu C, Smith BT, et al. COVID-19 workplace outbreaks by industry sector and their associated household transmission, Ontario, Canada, JanuaryJune, 2020 [preprint]. medRxiv 2020 Nov. 30. doi: 10.1101/2020.11.25.20239038.

4. Points to consider: public disclosure of outbreaks and cases of infectious diseases. Ottawa: Public Health Ethics Consultative Group (PHEGG) - Public Health Agency of Canada; modified 2021 Feb. 15. Available: https://www.canada.ca/en/public-health/ corporate/mandate/about-agency/external-advisory-bodies/list/public-health -ethics-consultative-group/points-consider-public-disclosure-outbreaks-cases -infectious-diseases.html (accessed 2021 Apr. 10).

5. Framework for ethical deliberation and decision-making in public health: a tool for public health practitioners, policy makers and decision-makers. Ottawa: Public Health Agency of Canada; 2017. Available: https://www.canada.ca/content/ dam/phac-aspc/documents/corporate/transparency/corporate-management -reporting/internal-audits/audit-reports/framework-ethical-deliberation-decision -making/pub-eng.pdf (accessed 2021 Apr. 10).

6. Public health ethics framework: a guide for use in response to the COVID-19 pandemic in Canada. Ottawa: Public Health Agency of Canada; modified 2021 Feb. 16. Available: https://www.canada.ca/en/public-health/services/ diseases/2019-novel-coronavirus-infection/canadas-reponse/ethics-framework -guide-use-response-covid-19-pandemic.html (accessed 2021 Apr. 10).

7. COVID-19 guidance: workplace outbreaks. Toronto: Ontario Ministry of Health; 2020. Available: https://www.health.gov.on.ca/en/pro/programs/publichealth/ coronavirus/docs/2019_workplace_outbreak_guidance.pdf (accessed 2021 Jan. 10).

8. Infection prevention and control disclosure protocol, 2019. Toronto: Ontario Ministry of Health and Long-Term Care; 2019 Feb. Available: https://www.health.gov. on.ca/en/pro/programs/publichealth/oph_standards/docs/protocols_guidelines /Infection_Prevention_and_Control_Disclosure_Protocol_2019_en.pdf (accessed 2021 May 1).

9. Toronto Public Health releases new COVID-19 workplace outbreak data and announces new measures for COVID-19 related workplace safety [news release]. Toronto: City of Toronto; 2021 Jan. 4. Available: https://www.toronto.ca/news/ toronto-public-health-releases-new-covid-19-workplace-outbreak-data-and -announces-new-measures-for-covid-19-related-workplace-safety/ (accessed 2021 Jan. 10).

10. Public Health implements changes to workplace/facility outbreak disclosures [news release]. Kitchener: Region of Waterloo; 2021 Mar. 17. Available: https://www. regionofwaterloo.ca/Modules/News/index.aspx?newsld=0c619fe6-8f0d-42af-8405 -24ee6cb8ba83\# (accessed 2021 Apr. 10).

11. Social stigma associated with COVID-19. Geneva: World Health Organization; updated 2020 Feb. 24. Available: https://www.who.int/docs/default-source/ coronaviruse/covid19-stigma-guide.pdf (accessed 2021 Jan. 10).

\section{Competing interests: None declared.}

This article has been peer reviewed.

Affiliations: Temerty Faculty of Medicine (Tran) and Dalla Lana School of Public Health (Loh), University of Toronto, Toronto, Ont.; Schulich School of Medicine and Dentistry (Zhong), Western University, London, Ont.; Peel Public Health, Mississauga (Loh), Mississauga, Ont.

Contributors: All of the authors contributed to the conception and design of the work, drafted the manuscript, revised it critically for important intellectual content, gave final approval of the version to be published and agreed to be accountable for all aspects of the work.

Content licence: This is an Open Access article distributed in accordance with the terms of the Creative Commons Attribution (CC BY-NCND 4.0) licence, which permits use, distribution and reproduction in any medium, provided that the original publication is properly cited, the use is noncommercial (i.e., research or educational use), and no modifications or adaptations are made. See: https://creativecommons.org/ licenses/by-nc-nd/4.0/

Correspondence to: Lawrence Loh, cmajgroup@cmaj.ca 